\title{
Unveiling the singular dynamics in the cosmic large-scale structure
}

\author{
Cornelius Rampf ${ }^{\oplus 1 \star}$, Uriel Frisch ${ }^{\circledR 1}$ and Oliver Hahn ${ }^{\oplus 1,2,3}$ \\ ${ }^{1}$ Université Côte d'Azur, Observatoire de la Côte d'Azur, CNRS, Laboratoire Lagrange, Boulevard de l'Observatoire, CS 34229 , 06304 Nice, France \\ ${ }^{2}$ Department of Astrophysics, University of Vienna, Türkenschanzstraße 17, 1180 Vienna, Austria \\ ${ }^{3}$ Department of Mathematics, University of Vienna, Oskar-Morgenstern-Platz 1, 1090 Vienna, Austria
}

Accepted 19 May 2021. Received 24 April 2021; in original form 29 January 2021

\begin{abstract}
It is known that the gravitational collapse of cold dark matter leads to infinite-density caustics that seed the primordial dark-matter halos in the large-scale structure. The development of these caustics begins, generically, as an almost one-dimensional phenomenon with the formation of pancakes. Focusing on the one-dimensional case, we identify a landscape of non-differentiable, and thus, singular features in the particle acceleration that emerge after the first crossing of particle trajectories. We complement our fully analytical studies by high-resolution simulations and find outstanding agreement, particularly shortly after the first crossing. We develop the methods in one space dimension but outline briefly the necessary steps for the 3D case.
\end{abstract}

Key words: instabilities - cosmology: theory - large-scale structure of Universe - dark matter

\section{INTRODUCTION}

A simple example of a singularity is when a function $\xi(\tau)$ has local behaviour $\propto\left(\tau-\tau_{1}\right)^{\lambda}$ around $\tau_{1}$, where $\lambda \notin \mathbb{N}$ is the singularity exponent. If $\lambda$ is a negative integer, then the singularity has a simple pole-like structure. If $\lambda$ is instead a positive non-integer number, then certain derivatives of $\xi(\tau)$ will blow up around $\tau_{1}$ and, as a result, $\xi(\tau)$ cannot be represented locally by a Taylor series.

In cosmology, the obvious singularities are density caustics that comprise the central building block for the cosmic large-scale structure. At the particle level, infinite-density caustics result from shell-crossing, the crossing of cold dark matter (CDM) trajectories. Once particles have crossed for the first time, the single-stream flow becomes multi-stream. Subsequently, secondary gravitational infall commences, inducing more shell-crossings that lead to a proliferation of streams, and eventually to virialized structures.

Some of the singularities were classified by Arnol'd (1980); Arnold et al. (1982), by exploiting an approximate nonlinear theory of gravitational instability, the Zel'dovich approximation (ZA; Zel'dovich 1970). However, singularities in derivatives of the particle trajectories, which we report here, remained undetected as the $\mathrm{ZA}$ is an acceleration-free model for nonlinear collapse, thereby being effectively blind to secondary gravitational infall.

Central to the analysis of Arnol'd (1980); Arnold et al. (1982), as well as ours, is the use of Lagrangian-coordinates approaches to gravitational instability that permit investigating singularities in a tractable manner. The ZA is the lowest-order Lagrangiancoordinates approximation to the cosmological fluid equations (the single-stream case of the Vlasov-Poisson equations). Furthermore, it is exact in 1D (Novikov 1969), as long as multi-stream flow has not yet appeared. Beyond 1D, higher-order approximations should

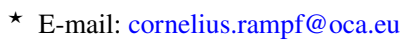

be incorporated, and the corresponding framework is dubbed Lagrangian perturbation theory (LPT; Buchert \& Götz 1987; Bouchet et al. 1992; Buchert 1992; Ehlers \& Buchert 1997; Bernardeau et al. 2002; Rampf \& Buchert 2012; Zheligovsky \& Frisch 2014). In LPT the displacement field is the only dynamical variable, which is expanded as a (temporal or spatial) Taylor series. Recently, the first nontrivial shell-crossing solutions in LPT have been identified (Rampf \& Frisch 2017; Rampf 2019), while numerical evidence of convergence of LPT in 3D at shell-crossing was given by Saga et al. (2018); Rampf \& Hahn (2021).

Nonetheless, the standard implementation of LPT cannot predict secondary gravitational infall, since it is based on a fluid description that does not incorporate multi-streaming. Instead, the multi-streaming evolution of CDM is governed by the VlasovPoisson equations. Following in the footsteps of Colombi (2015); Taruya \& Colombi (2017); Pietroni (2018), here we develop a Lagrangian-coordinates approach for Vlasov-Poisson and detect so far unknown singularities. These singularities comprise the intrinsic reason why standard perturbative techniques based on Taylor expansions break down at shell-crossing, while density singularities could be circumvented using suitable (Lagrangian) coordinates. For simplicity, we assume a 1D (spatial) Universe; the corresponding solutions play an important role in 3D cosmology, mainly because shell-crossings generically begin as almost $1 \mathrm{D}$ phenomena with the formation of pancakes (e.g. Melott \& Shandarin 1989). Notwithstanding, our theoretical tools are scalable to any dimensions with only mild modifications.

\section{SET-UP}

We denote by $q \mapsto x(q, \tau)$ the Lagrangian map from initial $(\tau=0)$ position $q$ to current position $x$ at time $\tau$, where $\tau$ is not the cos- 


\section{Rampf, Frisch \& Hahn}

mic time $t$ but is proportional to $t^{2 / 3}$. For simplicity, we assume a spatially flat and matter dominated Einstein-de Sitter (EdS) universe. We make use of comoving coordinates $x=r / a$, where $r$ is the proper space coordinate and $a$ the cosmic scale factor (for EdS: $a=\tau)$. The velocity is expressed in terms of the convective time derivative of the map, i.e., $v(x(q, \tau))=\partial_{\tau} x(q, \tau)=: \dot{x}(q, \tau)$. The Vlasov-Poisson equations for perfectly cold dark matter are

$\ddot{x}+\frac{3}{2 \tau} \dot{x}=-\frac{3}{2 \tau} \nabla_{x} \varphi, \quad \nabla_{x}^{2} \varphi=\frac{\delta(x(q, \tau))}{\tau}$,

where $\delta:=(\rho-\bar{\rho}) / \bar{\rho}$ is the dimensionless density contrast. Note that $\dot{x}=v=u / \partial_{t} a$ (where $u$ is the standard peculiar velocity) has units of lengths due to the use of a dimensionless time variable $\tau=a$; for simplicity, since no physical scales are introduced in an EdS universe, we set from here on all units to unity.

Both numerical N-body methods and theory aim to solve equations (1), however with at least one substantial difference, namely that in N-body methods the density contrast is determined by using an $\mathrm{N}$-particle approximation. By contrast, in theory one can determine the density using (Taylor \& Hamilton 1996)

$\delta(x(q, \tau))=\int \delta_{\mathrm{D}}\left[x(q, \tau)-x\left(q^{\prime}, \tau\right)\right] \mathrm{d} q^{\prime}-1$,

where " $\delta_{\mathrm{D}}$ " is the Dirac-delta.

Observe that equations (1) are invariant under the non-Galilean coordinate transformation $x \rightarrow x+\zeta_{0}(\tau)$, where $\zeta_{0}$ is an arbitrary function of time (Heckmann \& Schücking 1955; Ehlers \& Buchert 1997). In the present context we use this symmetry to enforce the following center-of-mass condition for the Lagrangian displacement field $\xi(q, \tau):=x(q, \tau)-q$ on the torus $\mathbb{T}$,

$\int_{\mathbb{T}} \xi\left(q^{\prime}, \tau\right) \mathrm{d} q^{\prime}=0, \quad \forall \tau>0$.

Note that periodic boundary conditions are assumed in this letter.

\section{SOLUTION STRATEGY AND INITIAL DATA}

Following a standard procedure (e.g. Bernardeau et al. 2002), equations (1) and (2) can be combined into a single equation by first taking the Eulerian (or Lagrangian) divergence of the former. Converting the derivatives according to $\left(\partial_{q} x\right) \partial_{x}=\partial_{q}$, we obtain

$\partial_{q} \Re_{\tau} \xi=-\frac{3}{2} F(x(q, \tau))$,

where $\mathfrak{R}_{\tau}=\tau^{2} \partial_{\tau}^{2}+(3 \tau / 2) \partial_{\tau}-3 / 2$ is the linear growth operator with eigenvalues +1 and $-3 / 2$, and

$F(x(q, \tau)):=\left(\partial_{q} x\right) \int \delta_{\mathrm{D}}\left[x(q, \tau)-x\left(q^{\prime}, \tau\right)\right] \mathrm{d} q^{\prime}-1$

is the effective multi-stream force ( $F=0$ in single-stream regions). See the supplementary material A1 for derivations. Integrating (4) in space from 0 to $q$, we obtain our main evolution equation

$\mathfrak{R}_{\tau}\left\{\xi(q, \tau)-\xi_{\mathrm{c}}(\tau)\right\}=-\frac{3}{2} S(x(q, \tau))$.

Here, $S(x(q, \tau)):=\int_{0}^{q} F\left(x\left(q^{\prime}, \tau\right)\right) \mathrm{d} q^{\prime}$ is the integrated multistreaming force, while $\xi_{\mathrm{c}}(\tau):=\xi(q=0, \tau)$ is a space-independent integration constant which, as we show, needs to be adjusted due to multi-streaming by virtue of (3), such that equations (1) and (6) agree with each other.
To solve equation (6), we provide growing-mode initial conditions at $\tau=0$. We specify, actually without loss of generality (see supplementary material A2), the initial velocity to be periodic,

$\dot{\xi}(q, \tau=0)=-\sin q+c \sin ^{4} q-\frac{6 c}{5} \sin ^{6} q=: v^{(\mathrm{ini})}$,

where $c$ is a free parameter, and we have added a counter term $\propto \sin ^{6} q$ to avoid trivial violations of (3). In the real Universe, the initial velocity is of random nature, which however may be locally expanded as $v^{\text {ini }}=-a q+b q^{3}+c q^{4}+\ldots$, where $a-c$ are free parameters that do not alter the nature of the reported singularities, and we have removed a quadratic term by a Galilean transformation. We will see shortly that such low-order truncations allow us to accurately determine the post-shell-crossing forces by analytical means. Note that the setting $c=0$, as effectively employed by Taruya \& Colombi (2017), enforces a perfectly point-symmetric collapse which we claim however is degenerate in a Universe with random initial conditions. Therefore we keep $c$ non-zero, but assume for simplicity that it is sufficiently small ( $c \lesssim 0.49$ ), ensuring that the location of the first shell-crossing, controlled by the minimum of $\partial_{q} v^{\text {(ini) }}$, occurs at the origin $q=0$ in our chosen coordinate system.

\section{SHELL-CROSSING SOLUTION}

In the single-stream regime the spatial integral in $F$ simplifies as there is only a single root $x(q, \tau)=x\left(q^{\prime}, \tau\right)$ that contributes to the integral, yielding $1 /\left(\partial_{q} x\right)$; thus $F=0$ and so does its integral, $S=0$. Hence, equation (6) reduces to $\Re_{\tau}\left\{\xi-\xi_{\mathrm{c}}\right\}=0$. Furthermore, due to the absence of asymmetries in the evolution equation, we have, by virtue of the center-of-mass condition (3), that $\xi_{\mathrm{c}}=0$. Thus, the evolution equation can be solved with the initial condition (7), and we recover the well-known Zel'dovich solution (Zel'dovich 1970)

$x_{\mathrm{ZA}}(q, \tau)=q+\tau v^{(\mathrm{ini})}(q)$.

This solution is only valid until the time of first shell-crossing, denoted with $\tau_{\star}$, that is when the particle trajectory loses its singlevaluedness and CDM enters into the multi-stream regime. For topological reasons (cf. Fig. 1), the first appearance of $\partial_{q} x_{\mathrm{ZA}}=0$ marks the first shell-crossing, which, as is well known, is accompanied with an infinite density (cf. equation 2):

$\delta\left(x_{\mathrm{ZA}}\left(q_{\star}, \tau_{\star}\right)\right)=\frac{1}{\left.\partial_{q} x_{\mathrm{ZA}}\left(q, \tau_{\star}\right)\right|_{q=q_{\star}}}-1=\infty$.

It is easily checked that for the considered initial conditions, the first shell-crossing occurs at $\tau_{\star}=1$ at $q=q_{\star}=0$, for $c \lesssim 0.49$.

\section{POST-SHELL-CROSSING DYNAMICS}

To make progress on the analysis after shell-crossing, we introduce an iterative scheme for (6) in which the evolution of the post-shellcrossing (PSC) displacement, $\xi_{\text {PSC }}$, is driven by an integrated force resulting from the Zel'dovich flow, i.e.,

$\Re_{\tau}\left\{\xi_{\mathrm{PSC}}(q, \tau)-\xi_{\mathrm{c}}(\tau)\right\}=-\frac{3}{2} S_{\mathrm{ZA}}(q, \tau)$

in the first iteration, where $S_{\mathrm{ZA}}(q, \tau):=\int_{0}^{q} F\left(x_{\mathrm{ZA}}\left(q^{\prime}, \tau\right)\right) \mathrm{d} q^{\prime}$. In the following we summarize the main technical steps to solve (10), while in-depth derivations and instructions for higher-order refinements are respectively provided in the supplementary material B and $\mathrm{C}$. We remark that our iterative scheme is related to the one 


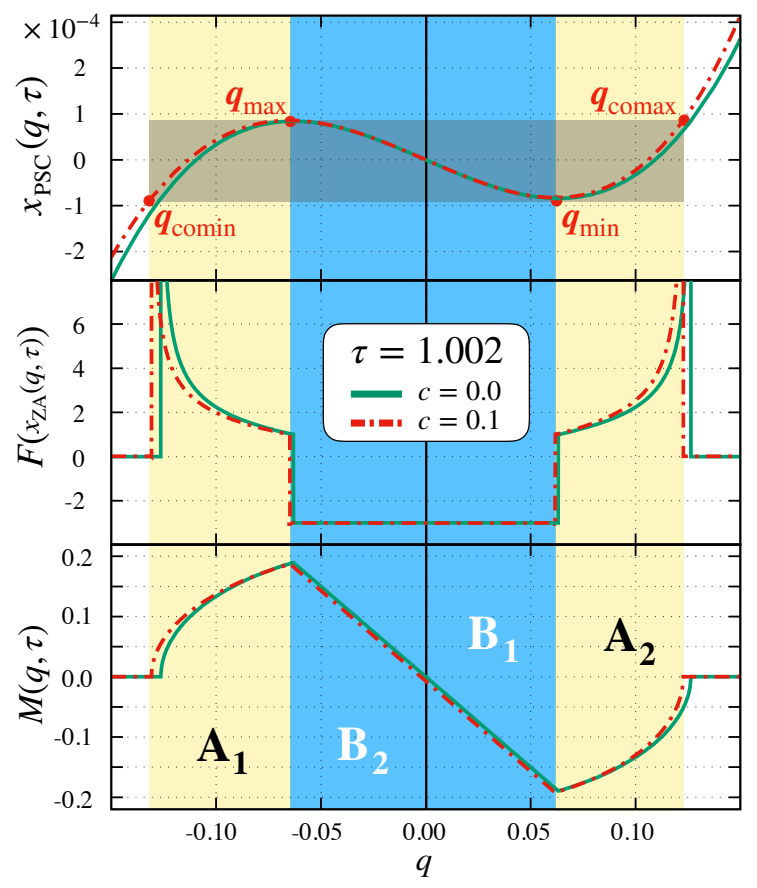

Figure 1. Shown is the post-shell-crossing map $x_{\mathrm{PSC}}=q+\xi_{\mathrm{PSC}}$ (top panel), the multi-streaming force $F$ (middle panel) and $M:=S_{\mathrm{ZA}}-S_{\mathrm{c}}$ (lower panel). All plots are evaluated after the first shell-crossing $\left(\tau_{\star}=1\right)$, namely at $\tau=1.002$. Green (solid) lines denote $c=0$, whereas red (dot-dashed) lines $c=0.1$. The grey (horizontally) shaded region marks the multi-streaming region (for $c=0.1$, which spans up the ascending $\left(\mathbf{A}_{\mathbf{1}, 2} ;\right.$ yellow-shaded) and the descending $\left(\mathbf{B}_{\mathbf{1}, \mathbf{2}} ;\right.$ blue-shaded $)$ multi-stream regimes. The single-stream regime $\left(\mathbf{S}_{\mathbf{1}, \mathbf{2}}\right)$ has no shading; in this context, note that due to the assumed periodicity, that $q \in[-\pi, \pi]$. The sharp non-differentiable features in $F$ and $M$, as well as the slight shift of $F$ and $M$ in the presence of nonzero $c$, are physical effects associated to singular behavior.

of Taruya \& Colombi (2017) but there are differences; see the supplementary material $\mathrm{D}$ for details.

We first need to solve for $S_{\mathrm{ZA}}$; for this, observe that the force (5) can be written as $F(x(q, \tau))=\int \partial_{q} \Theta\left(x(q, \tau)-x\left(q^{\prime}, \tau\right)\right) \mathrm{d} q^{\prime}-$ 1 , where $\Theta$ is the Heaviside step function. Therefore, the spatial integral of the force from 0 to $q$, within the $\mathrm{ZA}$, is simply

$S_{\mathrm{ZA}}=\int\left[\Theta\left(x_{\mathrm{ZA}}(q, \tau)-x_{\mathrm{ZA}}\left(q^{\prime}, \tau\right)\right)-\Theta\left(-x_{\mathrm{ZA}}\left(q^{\prime}, \tau\right)\right)\right] \mathrm{d} q^{\prime}-q$,

where we have used that $x_{\mathrm{ZA}}(q=0, \tau)=0$. Thus, solving for $S_{\mathrm{ZA}}$ amounts to finding the positions (roots) when the arguments of the $\Theta$ 's change their signs. Shortly after shell-crossing, and given that $c$ is small, both arguments of the $\Theta$ 's in equation (11) have three physical roots, implying that the flow has entered the three-stream regime (grey shading in Fig. 1).

Furthermore, the multi-streaming regime is still confined to small areas around $q=0=q_{\star}$, thus the positions of the three roots can be obtained by considering the low-order truncation $v^{\text {ini }} \approx$ $-q+q^{3} / 6+c q^{4}$ in $x_{\mathrm{ZA}}$ (higher-order terms do not change the nature of the reported singularities). For small $c$, the positions of the three roots are slightly shifted with respect to the $c=0$ case, which can be determined in perturbation theory, yielding

$S_{\mathrm{ZA}}=S_{\mathrm{c}}+\left\{\begin{array}{lrr}0 ; & 0 \leq \tau \leq \tau_{1} ; & \mathbf{S}_{\mathbf{1 , 2}} \\ -\operatorname{sign}(q) \sqrt{D(q, \tau)} ; & \tau_{1} \leq \tau \leq \tau_{2} ; & \mathbf{A}_{\mathbf{1}, \mathbf{2}} \\ -3 q-36 c(1-1 / \tau) ; & \tau \geq \tau_{2} ; & \mathbf{B}_{\mathbf{1}, \mathbf{2}}\end{array}\right.$ where, stemming from the $q=0$ part of (11),

$S_{\mathrm{c}}=36 c(1-1 / \tau) \Theta(\tau-1)$,

to first order in $c$. Here we have defined

$D(q, \tau)=24-3 q^{2}-24 / \tau+24 c q\left(3-q^{2}-3 / \tau\right)$,

as well as the space-dependent times

$\tau_{1}(q)=8 /\left(8-q^{2}-5 c q^{3}\right), \quad \tau_{2}(q)=2 /\left(2-q^{2}-8 c q^{3}\right)$.

Conversely, the Lagrangian positions can be expressed by $\tau_{1}$ and $\tau_{2}$, respectively leading to $q_{\mathrm{comin} / \mathrm{comax}}=\mp \sqrt{8(1-1 / \tau)}-20 c(1-$ $1 / \tau)$ and $q_{\min / \max }= \pm \sqrt{2(1-1 / \tau)}-8 c(1-1 / \tau)$, to first order in $c$. Also, we have $x\left(q_{\min / \max }\right)=x\left(q_{\text {comin/comax }}\right)$ by definition, where comin/comax stands for co-minimum/co-maximum.

For convenience, we have marked the positions $q_{\text {comin/comax }}$ and $q_{\min } / \max$ in Figure 1, where we also show the multi-streaming force $F\left(x_{\mathrm{ZA}}(q, \tau)\right)=\partial_{q} S_{\mathrm{ZA}}$ and the integral $M:=S_{\mathrm{ZA}}-S_{\mathrm{c}}$. Observe the appearance of several non-differential features in $F\left(x_{\mathrm{ZA}}(q, \tau)\right)$ and $S_{\mathrm{ZA}}$, indicating singularities; see the following section for a thorough analysis. We also note that the density is infinite at $q_{\min / \max }$, where $\left.\partial_{q} x(q)\right|_{q=q_{\min / \max }}=0$, which is wellknown; see e.g. Zel'dovich (1970); Arnold et al. (1982).

Let us first derive $\xi_{\mathrm{c}}(\tau)$, which is generally nonzero due to a forcing imbalance. For this we solve equation (10) in the $q=0$ case, which, using equations (12) and (3), reduces to $(\tau \geq 1)$

$\Re_{\tau} \xi_{\mathrm{c}}(\tau)=54 c(1-1 / \tau)$.

Supplemented with the boundary conditions at shell-crossing $\xi_{\mathrm{c}}(\tau=1)=0=\dot{\xi}_{\mathrm{c}}(\tau=1)$, we obtain for the particle that is initially at $q=0$ the following trajectory $(\tau \geq 1)$

$\xi_{\mathrm{c}}(\tau)=-\frac{18 c}{5}\left(10+8 \tau^{-3 / 2}-15 / \tau-3 \tau\right)$.

Thus, $\xi_{\mathrm{c}}$ is an effective time-dependent boost that switches on only after shell-crossing, which is clearly non-analytic behaviour; see section 6 for details. Some indications of that boost, for initial conditions of a distorted Gaussian shape, have been computed numerically in Pietroni (2018); see their Fig. 5. We note that the boost $\xi_{\mathrm{c}}$ does not only affect the $q=0$ particle, as shown below.

Having obtained $S_{\mathrm{ZA}}$ and $\xi_{\mathrm{c}}$, equation (10) can be straightforwardly solved by the method of variation of constants, $\xi_{\mathrm{PSC}}=$ $\lambda(\tau) \tau+\mu(\tau) \tau^{-3 / 2}$, with boundary conditions provided at shellcrossing, i.e., $\xi_{\mathrm{PSC}}(\tau=1)=\dot{\xi}_{\mathrm{PSC}}(\tau=1)=v^{(\text {ini) }}$, where $v^{(\text {ini })}$ is given in equation (7). See the supplementary material B2 for calculational details. We finally obtain the post-shell-crossing displacement

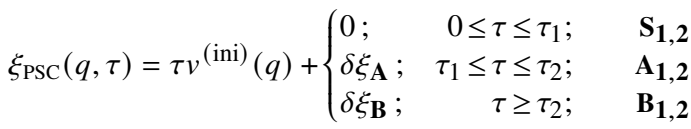

where

$\delta \xi_{\mathbf{A}}=\frac{\operatorname{sign}(q)}{180} \frac{D^{5 / 2}(q, \tau) \tau}{8-q^{2}+c q\left(48-11 q^{2}\right)}$,

$\delta \xi_{\mathbf{B}}=\xi_{\mathrm{c}}(\tau)-3 q+\frac{9 q \tau}{20}\left(4-q^{2}\right)+\frac{48}{5} \sqrt{\frac{2}{2-q^{2}}} \frac{q \tau^{-3 / 2}}{8-q^{2}}$

$+\frac{9 c}{20}\left[64 \tau^{-3 / 2}-\tau q^{4}-\frac{16 q^{4}\left(3 q^{2}-4\right) \tau^{-3 / 2}}{\left(1-q^{2} / 2\right)^{3 / 2}\left(q^{2}-8\right)^{2}}-96 \tau_{2}^{1 / 2} / \tau^{3 / 2}\right.$

$\left.+32 \tau_{2}^{3 / 2} / \tau^{3 / 2}-24 \tau\left(1-1 / \tau_{2}\right)^{2}\right]$.

The resulting map $x_{\mathrm{PSC}}(q, \tau)=q+\xi_{\mathrm{PSC}}(q, \tau)$ is shown in the top 

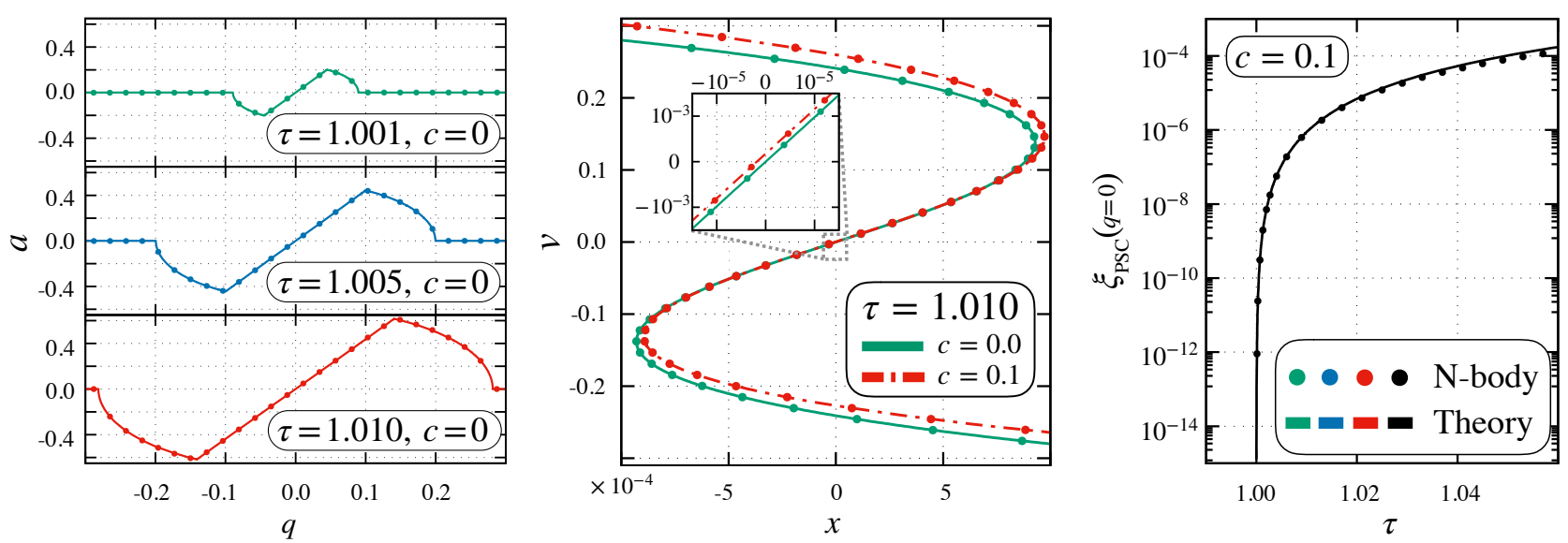

Figure 2. Results of theory (solid or dot-dashed lines) against numerical simulations (dotted; only every 30 th data point shown) shortly after the first shellcrossing $(\tau=1)$. The left panel shows the acceleration $a:=\ddot{x}_{\text {PSC }}=\ddot{\xi}_{\text {PSC }}$ as a function of the initial position $q$ for $c=0$, displaying four non-differentiable sharp features and thereby unveiling singular dynamics (theory and numerics agree almost perfectly). The central panel shows the phase-space shortly after shell-crossing, for $c=0$ (green) and $c=0.1$ (red); it involves a slight shift of the particles in the center of the multi-stream regime for $c \neq 0$ when the force is asymmetrical. The rightmost panel shows the same effect as a function of time, for the particle that was initially at $q=0$. The results depicted here cannot be predicted by the ZA; see the supplementary material E for details, including comparisons between ZA and PSC theory as well as late-time results.

panel of Fig. 1. For convenience, in that figure we have shaded the ascending ( $\mathbf{A}_{\mathbf{1}, \mathbf{2}}$, yellow) and descending $\left(\mathbf{B}_{\mathbf{1 , 2}}\right.$, blue) multi-stream branches, while single-stream regions $\left(\mathbf{S}_{\mathbf{1}, \mathbf{2}}\right)$ have no shading.

\section{SINGULARITIES IN SPACE AND TIME}

Starting from the instant of first shell-crossing, some CDM particles are directly exposed to infinite densities at their current positions. We show that the displacements of those exposed particles cannot be represented by convergent spatial or temporal Taylor series, which directly implies that standard perturbative techniques break down. For this we identify the rational singularity exponents $\lambda$, which indicate the integer perturbation order $n:=\lceil\lambda\rceil$ at which the $n$th derivative of the displacement, i.e., its Taylor coefficient, blows up.

Singularities in the displacement (18) could originate from two distinct sources, either by (a) explicit non-analytic features within the piecewise defined branches of the map, and/or by (b) discontinuities that arise when "gluing" the branches together. Related to (a), it is easily checked that the only non-analyticity within the branches arises when $D(q, \tau)$ vanishes (equation 14), which occurs at $\tau=\tau_{1}$. Related to (b), two other singularities are revealed at $\tau_{2}$.

To identify the (a)-type singularity it suffices to limit ourselves to $c=0$. Indeed, a vanishing discriminant $D$ is achieved by freezing the space dependence in $D$ and investigating small discrepancies $\delta \tau>0$ around $\tau_{1}$ from above. Taylor-expanding the term with the discriminant around $\delta \tau$ then leads to a $\delta \tau^{5 / 2}$ singularity. Thus, the displacement behaves locally as $\propto\left(\tau-\tau_{1}\right)^{5 / 2}$, implying that its third time derivative blows up at $\tau_{1}$. Similarly, by freezing the time and varying $q$, one finds spatial singularities at $q_{\text {comax } / \text { comin with }}$ exponent $5 / 2$. Physically, what happens is that a spectator particle near $q_{\text {comax }}$ comin crosses a caustic at the current position.

The (b)-type singularities are expected to occur at $\tau_{2}$. One of those singularities stems from glueing the ascending and descending multi-stream branches together, for which purpose it suffices to set $c=0$. Taylor-expanding around small temporal discrepancies near $\tau_{2}$, we find that the third-order time derivative flips sign, indicating that the third derivative of $\xi$ is discontinuous, thereby marking a singularity of $\xi \sim\left(\tau-\tau_{2}\right)^{3} \theta\left(\tau_{2}-\tau\right)$. Similarly, we find that the third spatial derivative of the map is also discontinuous, thus implying spatial singularities near $q_{\min / \max }$ of exponent 3 .

Lastly, a nontrivial singularity originates from $\xi_{\mathrm{c}}$ (equation 17), which is driven by a force imbalance (from $c \neq 0$ in equation 7); this manifests itself through the loss of analyticity at the first shellcrossing, thereby resulting in a dynamical phase transition due to a broken symmetry in the multi-streaming force. Indeed, the boost $\xi_{\mathrm{c}}$ "switches" from off to on $\left(\propto \delta \tau^{3}\right)$ once multi-streaming develops (right panel in Fig. 2). Consequently, a particle that is initially at $q=0$ will remain there until the first shell-crossing at $\tau=1$, but then the first time-derivative of its acceleration receives a non-analytic contribution, jumping from 0 to a finite number.

The (a)-type and first (b)-type singularities are shown in the first panel of Fig. $2(c=0)$, while the second (b)-type singularity is displayed in the third panel, for $c=0.1$. We confront these findings against $1 D$ numerical ( $N$-body) simulations that determine the exact particle forces using an efficient sorting algorithm (for a similar implementation however in a non-periodic set-up, see Colombi \& Touma 2014). The results of our simulations are marked by dots in Fig. 2. The respective 1D code, which we make publicly available, ${ }^{1}$ solves equations (1) with a symplectic time-integrating scheme. Initial conditions are provided by the ZA using (7) in a periodic box for $x, q \in[-\pi, \pi]$ (physical units can be trivially restored in the code if needed). Runs were performed with $10^{4} \mathrm{CDM}$ particles and time-steps, though for detecting the singularity stemming from $\xi_{\mathrm{c}}$, temporal and spatial resolutions of up to $10^{5}$ have been used.

\section{CONCLUSIONS}

Associated to the known shell-crossing singularities in the colddark-matter density, we have identified three non-differentiable acceleration features. These features imply non-analytic behavior as derivatives in the phase-space blow up; therefore, any analytical technique that solves for the CDM dynamics by using temporal or spatial perturbative expansions will break down at shell-crossing.

\footnotetext{
1 https://bitbucket.org/ohahn/cosmo_sim_1d
} 
Two of those singularities are of local origin and appear either when particles enter a multi-streaming region, or when particles are directly involved in the density caustic (Fig. 1 and first panel in Fig. 2). Yet, a third singularity is of global origin emerging from a boost in the theory, $\xi_{\mathrm{c}}(\tau)$, needed to establish momentum conservation in asymmetric collapse scenarios (second and third panel in Fig. 2). In numerical simulations, by contrast, $\xi_{\mathrm{c}}(\tau)$ appears implicitly by preserving the fundamental conservation laws for Vlasov-Poisson.

Why are the reported singularities relatively so weak? VlasovPoisson is intimately linked to a Hamiltonian formulation and is therefore constrained by Liouville's theorem (phase-space incompressibility), which guarantees that perfectly cold dark matter is devoid of any disruptions in the position and velocity space (central panel in Fig. 2). One can show that in the generic case of initial data, which do not possess parity invariance $(c \neq 0$ in equation 7$)$, any attempt to construct solutions that ignore $\xi_{\mathrm{c}}(\tau)$ will lead to severe disruptions of the phase-space, thereby violating phase-space incompressibility.

A straightforward yet challenging extension to our work is to exploit the singularity theory in quasi-1D, where departures from 1D are perturbatively small, thus providing a bookkeeping parameter (cf. Rampf \& Frisch 2017). Generalizations to 3D are feasible as well, by using higher-order LPT (Zheligovsky \& Frisch 2014; Rampf et al. 2015; Matsubara 2015; Rampf \& Hahn 2021) and providing boundary conditions at shell-crossing, especially for trigonometric initial conditions, where fast Fourier transforms can be avoided (cf. Saga et al. 2018). In the present work, we have provided the stepping stones for such avenues; indeed, equations (1)-(3) are trivially generalized to arbitrary dimensions.

A full-fledged theory for the large-scale structure has the potential to advance its theoretical predictions. For example, the theory can determine inputs for heavily used effective theories of the large-scale structure (e.g. Baumann et al. 2012; Porto et al. 2014). Indeed, such effective theories incorporate shell-crossing and multistreaming effects through counter terms (with a priori unknown time dependence), which are usually estimated from N-body simulations.

Finally, the Vlasov-Poisson equation applies also when the gravitational field gets replaced by an electrostatic (repulsive) field. One direct application of our theory is the bump-on-tail instability in which a beam of charged particles moves in a background neutral plasma (e.g. O'Neil et al. 1971; Lesur \& Diamond 2013; Escande et al. 2018). Indeed, apart from minor adaptions (e.g., sign change of charge), our evolution equations are identical with the one of O'Neil et al. (1971) in the continuous limit. Generalizations to multiple cold beams or to the warm case are straightforward too (see e.g. Carlevaro et al. 2015), and could provide significant insights to the development of instabilities in plasma physics.

\section{ACKNOWLEDGEMENTS}

We thank Patrick Diamond, Massimo Pietroni, Zachary Slepian and Matias Zaldarriaga for useful discussions. CR acknowledges funding from the People Programme (Marie Skłodowska-Curie Actions) of the European Union's Horizon 2020 Programme under Grant Agreement No. 795707 (COSMO-BLOW-UP). UF acknowledges financial support from the Université de la Côte d'Azur under Grant Agreement No. ANR-15-IDEX-01 (2018-2019). OH acknowledges funding from the European Research Council (ERC) under the European Union's Horizon 2020 research and innovation programme (Grant Agreement No. 679145, project 'COSMO-SIMS').

\section{DATA AVAILABILITY}

The code to perform N-body simulations in 1D is freely available at https://bitbucket.org/ohahn/cosmo_sim_1d. The data underlying this article will be shared on reasonable request to the corresponding author.

\section{REFERENCES}

Arnol'd V. I., 1980, Mathematical Methods of Classical Mechanics. Springer, New York

Arnold V. I., Shandarin S. F., Zeldovich I. B., 1982, Geophysical and Astrophysical Fluid Dynamics, 20, 111

Baumann D., Nicolis A., Senatore L., Zaldarriaga M., 2012, J. Cosmology Astropart. Phys., 2012, 051

Bernardeau F., Colombi S., Gaztañaga E., Scoccimarro R., 2002, Phys. Rep., 367,1

Bouchet F. R., Juszkiewicz R., Colombi S., Pellat R., 1992, ApJ, 394, L5

Buchert T., 1992, MNRAS, 254, 729

Buchert T., Götz G., 1987, Journal of Mathematical Physics, 28, 2714

Carlevaro N., Falessi M. V., Montani G., Zonca F., 2015, J. Plasma Phys., 81,495810515

Colombi S., 2015, MNRAS, 446, 2902

Colombi S., Touma J., 2014, MNRAS, 441, 2414

Ehlers J., Buchert T., 1997, General Relativity and Gravitation, 29, 733

Escande D. F., Bénisti D., Elskens Y., Zarzoso D., Doveil F., 2018, Rev. Mod. Plasma Phys., 2, 9

Heckmann O., Schücking E., 1955, Z. Astrophys., 38, 95

Lesur M., Diamond P. H., 2013, Phys. Rev. E, 87, 031101

Matsubara T., 2015, Phys. Rev. D, 92, 023534

Melott A. L., Shandarin S. F., 1989, ApJ, 343, 26

Novikov E. A., 1969, Sov. Phys. JETP, 30, 512

O’Neil T. M., Winfrey J. H., Malmberg J. H., 1971, Phys. Fluids, 14, 1204

Pietroni M., 2018, J. Cosmology Astropart. Phys., 2018, 028

Porto R. A., Senatore L., Zaldarriaga M., 2014, J. Cosmology Astropart. Phys., 2014, 022

Rampf C., 2019, MNRAS, 484, 5223

Rampf C., Buchert T., 2012, J. Cosmology Astropart. Phys., 2012, 021

Rampf C., Frisch U., 2017, MNRAS, 471, 671

Rampf C., Hahn O., 2021, MNRAS, 501, L71

Rampf C., Villone B., Frisch U., 2015, MNRAS, 452, 1421

Saga S., Taruya A., Colombi S., 2018, Phys. Rev. Lett., 121, 241302

Taruya A., Colombi S., 2017, MNRAS, 470, 4858

Taylor A. N., Hamilton A. J. S., 1996, MNRAS, 282, 767

Uhlemann C., Rampf C., Gosenca M., Hahn O., 2019, Phys. Rev. D, 99, 083524

Zel'dovich Ya. B., 1970, A\&A, 500, 13

Zheligovsky V., Frisch U., 2014, J. Fluid Mech., 749, 404

\section{SUPPORTING INFORMATION}

Supplementary data are available at MNRASL online.

Figure S1. The boost $\xi_{\mathrm{c}}(\tau)$ for different counter terms.

Figure S2. Analysis of the involved integrands in $S_{\mathrm{ZA}}$.

Figure S3. The multi-streaming force at the PSC refinement level.

Figure S4. Late-time evolution of the boost $\xi_{\mathrm{c}}(\tau)$.

Figure S5. Late-time evolution of the phase-space with $c=0.1$.

Figure S6. Late-time evolution of the phase-space with $c=0.0$.

Please note: Oxford University Press is not responsible for the content or functionality of any supporting materials supplied by the authors. Any queries (other than missing material) should be directed to the corresponding author for the article.

This paper has been typeset from a $\mathrm{T}_{\mathrm{E}} \mathrm{X} / \mathrm{L} \mathrm{T}_{\mathrm{E}} \mathrm{X}$ file prepared by the authors. 


\section{SUPPLEMENTARY MATERIAL}

\section{SUPPLEMENTARY MATERIAL A: EVOLUTION EQUATIONS AND CHOICE OF INITIAL DATA}

In the following we address both the aspects of our chosen theoretical starting point (section A1) as well as outline the arguments that support the generality of findings (section A2).

\section{A1 Derivation of main evolution equation}

Here we provide details to the derivation of our main evolution equation (4). We begin with the standard Vlasov-Poisson equations for dark matter, which we repeat here for convenience (see e.g. Uhlemann et al. 2019)

$\ddot{x}+\frac{3}{2 \tau} \dot{x}=-\frac{3}{2 \tau} \nabla_{x} \varphi$,

$\nabla_{x}^{2} \varphi=\frac{\delta(x(q, \tau))}{\tau}$,

where

$\delta(x(q, \tau))=\int \delta_{\mathrm{D}}\left[x(q, \tau)-x\left(q^{\prime}, \tau\right)\right] \mathrm{d} q^{\prime}-1$.

To proceed, we take the Eulerian divergence of equation (A1a), which allows us to express its right-hand side in terms of the Poisson equation, leading to

$\nabla_{x}\left(\ddot{x}+\frac{3}{2 \tau} \dot{x}\right)=-\frac{3}{2 \tau^{2}}\left[\int \delta_{\mathrm{D}}\left[x(q, \tau)-x\left(q^{\prime}, \tau\right)\right] \mathrm{d} q^{\prime}-1\right]$.

Multiplying the last equation by $\tau^{2} \partial_{q} x$ and converting the Eulerian derivative according to $\left(\partial_{q} x\right) \nabla_{x}=\partial_{q}$, we obtain

$\partial_{q}\left(\tau^{2} \ddot{x}+\frac{3 \tau}{2} \dot{x}\right)=-\frac{3}{2} F(x(q, \tau))+\frac{3}{2}\left(\partial_{q} x-1\right)$,

where we have identified the effective multi-streaming force

$F(x(q, \tau))=-1+\left(\partial_{q} x\right) \int \delta_{\mathrm{D}}\left[x(q, \tau)-x\left(q^{\prime}, \tau\right)\right] \mathrm{d} q^{\prime}$.

Finally, we express in equation (A3) the Lagrangian map in terms of the displacement $\xi=x-q$, which leads to our main evolution equation

$\partial_{q} \Re_{\tau} \xi=-\frac{3}{2} F$,

where $\Re_{\tau}=\tau^{2} \partial_{\tau}^{2}+(3 \tau / 2) \partial_{\tau}-3 / 2$. We note that the Lagrangian space derivative commutes with the Lagrangian time derivative.

One particularly convenient feature of equation (A5) is that, in singlestream regions for which $F=0$, it trivially integrates to the standard differential equation for the Zel'dovich displacement (cf. section 4 in the main text). This feature allows to evolve all particles in single-stream regions using the Zel'dovich displacement, as well as providing accurate boundary conditions once particles are about to enter multi-streaming regions.

As regards to the computations of the multi-streaming force, equation (A4) involves a spatial integral over a Dirac delta which, upon spatial integration, can be converted into a Heaviside step-function $\Theta$. As we elucidate in detail in App. B, determining the integrated multi-streaming force using $\Theta$ 's amounts to a significant simplification. This is important for the present considerations, since integrations can be nontrivial due to the nonanalytic nature of the force in multi-streaming regions.

\section{A2 Initial conditions and generality of findings}

In the present paper we choose for the initial peculiar velocity

$v^{(\text {ini) }}=-\sin q+c \sin ^{4} q-\frac{6 c}{5} \sin ^{6} q$,

where we assume that $c$ is a small parameter. Although this initial velocity appears to be quite specific, we show in the following that our choice of initial data is actually generic, in the sense that the nature of the reported singularities does not change for similar choices of initial data (modulus trivial changes in prefactors etc.).

First of all, we use smooth periodic initial data as they are in accordance with the known observational fact of the random nature of primordial
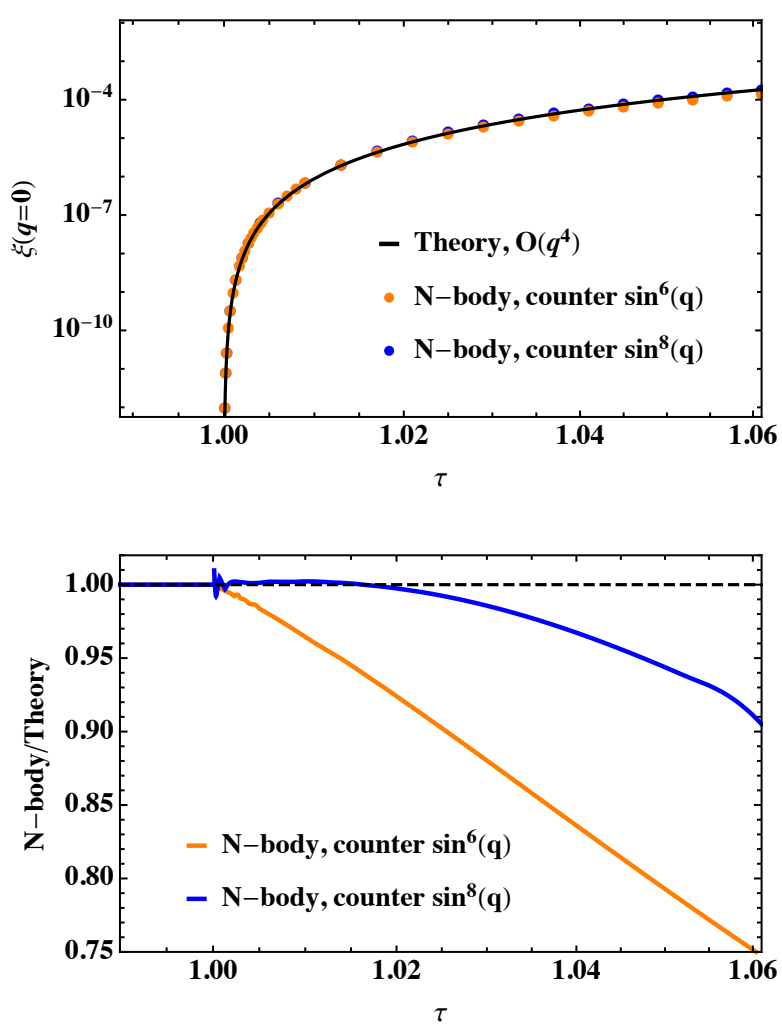

Figure S1. The late time-effect of adding higher-order counter terms in the initial data on the boost $\xi_{\mathrm{c}}(\tau)=\xi(q=0, \tau)$, for $c=0.1$. Top: The solid line denotes our theory results, while the orange [blue] dots denote simulation results using equation (A6) [equation (A8)] for the initial data. Bottom: Same as above but shown is the ratio N-body/theory. We note that the deviations in the ratio shortly after $\tau=\tau_{\star}=1$ are due to limited resolution in the numerical simulation.

density fluctuations (with vanishing mean). We remark that non-smooth initial conditions (such as logarithms) would imply singular initial conditions, whereas it is our goal here to see whether smooth initial conditions lead eventually to singularities.

At the technical level for detecting the singularities, we need to determine the particle forces shortly after shell-crossing to sufficient accuracy. For this it is crucial to notice that the spatial width of the multi-streaming region is very narrow shortly after shell-crossing, implying that low-order spatial truncations of the initial data are fully meaningful (in catastrophe theory, this is called a 'normal form' approach). Thus, to determine the force shortly after shell-crossing, it suffices to approximate (A6) with the spatial expansion

$v^{(\text {ini) }}=-q+\frac{q^{3}}{3 !}+c q^{4}+H . O . T .$,

where H.O.T. stands for higher-order terms; see Appendix C for higherorder refinements. We remark here that we exclude terms $\sim q^{2}$ in (A7) (e.g., stemming from a cosine in equation A6); indeed, their effect is boosting the system by a constant velocity, which can be removed by a Galilean transformation. Therefore, while the precise time and location of the first shell-crossing might change with such a new term, no new physics is added and thus, we can safely ignore such terms.

Our choice of initial data is fully generic also in the sense that one could decorate various numerical coefficients in front of the spatial terms appearing in the normal form (A7), which would not alter the nature of the reported singularities (but could clutter the analytical expressions).

Finally, the nature of the singularity related to the boost is unchanged, even if one employs different counter-terms in the initial velocity. This is 
illustrated in Fig. S1, where we show the particle trajectory at $q=0$ at times after shell-crossing, comparing the effects from different initial data while setting $c=0.1$. Specifically, the red dots and black line display results from using (A6) as the initial data, while for the blue dotes we use

$\tilde{v}^{(\text {ini) }}=-\sin q+c \sin ^{4} q-\frac{48 c}{35} \sin ^{8} q$

instead. As seen in Fig. S1, until shortly after shell-crossing, the depicted trajectory for the two sets of initial data coincide to good accuracy, especially for the initial data that includes the counter term $\sim c q^{8}$. Only at much later times the trajectories begin to differ, which is expected from theory grounds: at those late times the multi-stream regime has substantially gained on spatial width, where the counter terms in the initial data have increasing impact.

\section{SUPPLEMENTARY MATERIAL B: MULTI-STREAMING FORCE AND POST-SHELL-CROSSING DISPLACEMENT}

Here we provide calculational details about how the multi-streaming force $F$ (defined around equation 4), its spatial integral $S=\int F\left(q^{\prime}\right) \mathrm{d} q^{\prime}$ and the resulting post-shell-crossing displacement $\xi$ PSC are determined within the first PSC iteration, i.e., when the force, shortly after shell-crossing, is estimated using the Zel'dovich approximation.

We begin with the definition of the ZA force for which we approximate $x(q, \tau) \simeq x_{\mathrm{ZA}}(q, \tau)$ in $F$ (this approximation is exact until shell-crossing and approximative shortly after), i.e.,

$F\left(x_{\mathrm{ZA}}(q, \tau)\right)=\left(\partial_{q} x_{\mathrm{ZA}}\right) \int \delta_{\mathrm{D}}\left[x_{\mathrm{ZA}}(q, \tau)-x_{\mathrm{ZA}}\left(q^{\prime}, \tau\right)\right] \mathrm{d} q^{\prime}-1$,

where $x_{\mathrm{ZA}}(q, \tau)=q+\tau v^{(\text {ini) }}$, with

$v^{(\text {ini) }}=-\sin q+c \sin ^{4} q-\frac{6 c}{5} \sin ^{6} q$.

From equation (B1) it is clear that one could evaluate the density locally, namely by exploiting the composition property of the Dirac-delta, that for any function $x(q)$, we have $\delta_{\mathrm{D}}(x(q))=\sum_{i} \delta_{\mathrm{D}}\left(q-q_{i}\right) /\left|\partial_{q} x\left(q_{i}\right)\right|$, where $q_{i}$ is the $i$ th root of $x(q)$.

Alternatively, and as done in the main text, we can write equation (B1) equivalently as

$F\left(x_{\mathrm{ZA}}(q, \tau)\right)=\int \partial_{q} \Theta\left[x_{\mathrm{ZA}}(q, \tau)-x_{\mathrm{ZA}}\left(q^{\prime}, \tau\right)\right] \mathrm{d} q^{\prime}-1$,

which can be trivially integrated to $S_{\mathrm{ZA}}=\int_{0}^{q} F\left(x_{\mathrm{ZA}}\left(q^{\prime}, \tau\right)\right) \mathrm{d} q^{\prime}$, yielding

$S_{\mathrm{ZA}}=\int\left[\Theta\left(x_{\mathrm{ZA}}(q, \tau)-x_{\mathrm{ZA}}\left(q^{\prime}, \tau\right)\right)-\Theta\left(-x_{\mathrm{ZA}}\left(q^{\prime}, \tau\right)\right)\right] \mathrm{d} q^{\prime}-q$.

Using the step-function, $\Theta$, instead of the Dirac-delta simplifies the calculations significantly, especially when determining the boost $\xi_{\mathrm{c}}$. Indeed, the boost arises from global constraints related to the center-of-mass condition (3), which can be easily incorporated in (B4) by integration over the torus. By contrast, solving for the boost $\xi_{\mathrm{c}}$ in the local "Dirac-delta approach" is non-trivial: it can be done by considering the spatial average of $\xi$ PSC or, equivalently, by a weighted spatial average of its evolution equation. Nonetheless, we have explicitly verified that our final results coincide, whether one uses the Dirac-delta or the Heaviside approach.

In App. B1 we derive directly $S_{\mathrm{ZA}}$ by solving (B4), from which one can easily obtain $F\left(x_{\mathrm{ZA}}(q, \tau)\right)=\partial_{q} S_{\mathrm{ZA}}$. Then, in App. B2 we determine the post-shell-crossing displacement.

\section{B1 Solving for the multi-streaming force}

It is convenient to split up equation (B4) into two distinguished terms, i.e., $S_{\mathrm{ZA}}:=S_{\mathrm{ZA}, 1}+S_{\mathrm{ZA}, 2}$, with

$S_{\mathrm{ZA}, 1}=-\int \Theta\left(-x_{\mathrm{ZA}}\left(q^{\prime}, \tau\right)\right) \mathrm{d} q^{\prime}$,

$S_{\mathrm{ZA}, 2}=\int \Theta\left(x_{\mathrm{ZA}}(q, \tau)-x_{\mathrm{ZA}}\left(q^{\prime}, \tau\right)\right) \mathrm{d} q^{\prime}-q$.
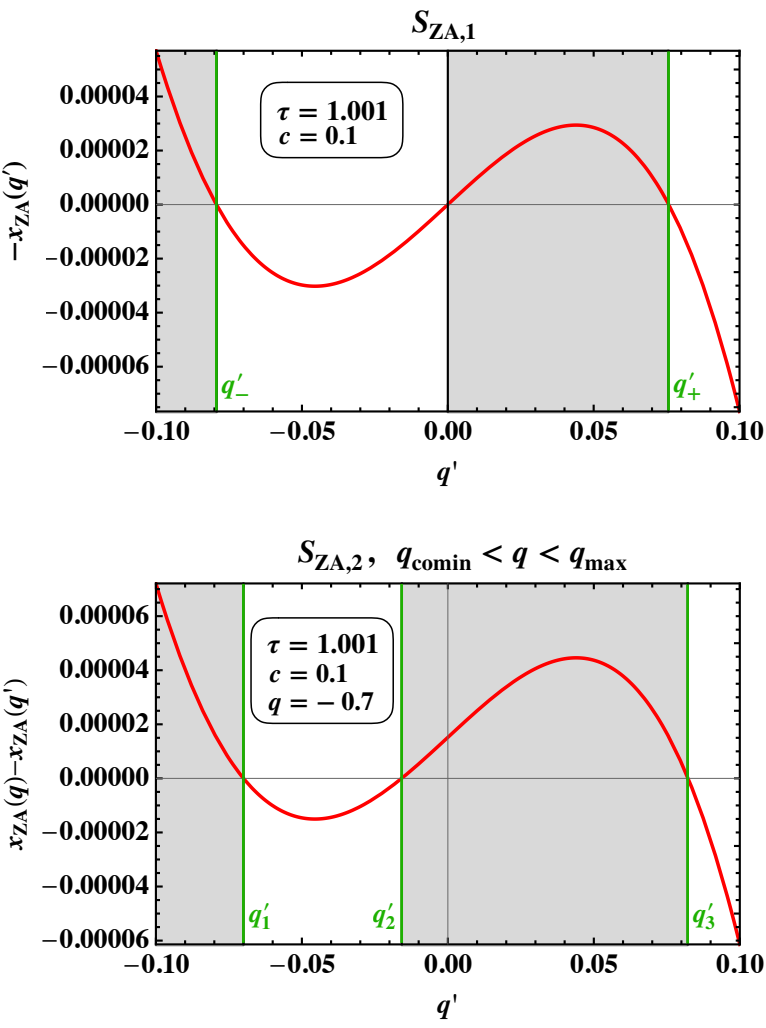

Figure S2. Shown are the arguments of the Heaviside functions appearing in the spatial integrals of $S_{\mathrm{ZA}}$ according to equation (B5), shortly after shellcrossing and for $c=0.1$. Top: $S_{\mathrm{ZA}, 1}$, which does not depend on $q$. Bottom: $S_{\mathrm{ZA}, 2}$ for the case when $q_{\text {comin }}<q<q_{\max }$.

Before shell-crossing, solving these equations is trivial, as both Heaviside functions have only one root. Thus, in the single-stream regime, the integration over the torus leads to $S_{\mathrm{ZA}, 1}=-\pi$ and $S_{\mathrm{ZA}, 2}=+\pi$; thus $S_{\mathrm{ZA}}=0$.

After the first shell-crossing, for sufficiently small $c$, which we assume throughout this work, there are Eulerian locations with three fluid streams (see Fig. 1), meaning that at such locations the arguments of the Heaviside functions in (B5) have each three roots. Furthermore, for given initial data and for short times after shell-crossing, the three-stream regime is confined to small spatial areas around $q=0$ (i.e., close to the Lagrangian locations where the first shell-crossing occurred). This implies that, to find the three roots shortly after shell-crossing, it suffices to consider the low-order spatial truncation (i.e., the normal form) of the initial data (B2), which is

$v^{(\text {ini) }}=-q+q^{3} / 6+c q^{4}+H \cdot O \cdot T .$,

to be used in the Zel'dovich map $x_{\mathrm{ZA}}=q+\tau v^{(\text {ini) }}$. Furthermore, since $c$ is assumed to be small, the effect of the term $c q^{4}$ in (B6) is to shift the positions of some of the roots; in the following we will determine this effect using linear perturbation theory.

We begin with solving for $S_{\mathrm{ZA}, 1}$ according to equation (B5a), which has the integrand $\Theta\left(-x_{\mathrm{ZA}}\left(q^{\prime}, \tau\right)\right)$, with its argument shown in the upper panel of Fig. S2. Shortly after shell-crossing and for vanishing $c$, the argument $-x_{\mathrm{ZA}}\left(q^{\prime}, \tau\right)$ has the three roots

$q^{\prime}=0, \quad \bar{q}_{ \pm}^{\prime}= \pm \sqrt{6(1-1 / \tau)}$,

where the overbar denotes the $c=0$ case. To include the effect of $c$, we set $q_{ \pm}^{\prime}=\bar{q}_{ \pm}^{\prime}+\delta q_{ \pm}$,

where $\delta q_{ \pm}=O(c)$, from which one easily finds that

$q_{ \pm}^{\prime}= \pm \sqrt{6(1-1 / \tau)}-18 c(1-1 / \tau)+O\left(c^{2}\right)$.

These roots are also shown in the upper panel of Fig. S2 as vertical green lines, while the grey shading indicates the regime where $-x_{\mathrm{ZA}}\left(q^{\prime}, \tau\right)$ is 
positive. From this one then finds that

$S_{\mathrm{ZA}, 1}=-\pi+ \begin{cases}0 ; & \tau<1 \\ 36 c(1-1 / \tau)+O\left(c^{2}\right) ; & \tau \geq 1\end{cases}$

Next we consider $S_{\mathrm{ZA}, 2}$, which involves an integral over a Heaviside function with argument $x_{\mathrm{ZA}}(q, \tau)-x_{\mathrm{ZA}}\left(q^{\prime}, \tau\right)$. In contrast to the previous calculation, the integral argument depends on $q$. Specifically, shortly after shell-crossing, solving $x_{\mathrm{ZA}}(q, \tau)-x_{\mathrm{ZA}}\left(\bar{q}^{\prime}, \tau\right)=0$ is, to leading order,

$\left(q-q^{\prime}\right)\left[q^{\prime 2}+q q^{\prime}+q^{2}+6 / \tau-6+6 c\left(q+q^{\prime}\right)\left(q^{2}+q^{\prime 2}\right)\right]=0$.

For vanishing $c$, the three roots of this equation are

$\bar{q}_{1}^{\prime}=q, \quad \bar{q}_{2,3}^{\prime}=\frac{-q \pm \sqrt{\bar{D}(q, \tau)}}{2}$,

where $\bar{D}(q, \tau)=24-3 q^{2}-24 / \tau$. Similarly as above, the effect of small $c$ can be incorporated using perturbation theory, leading to

$q_{1}^{\prime}=q, \quad q_{2,3}^{\prime}=\frac{1}{2}(-q-36 c(1-1 / \tau) \pm \sqrt{D(q, \tau)})$

to first order in $c$, where

$D(q, \tau):=24-3 q^{2}-24 / \tau+24 c q\left(3-q^{2}-3 / \tau\right)$.

In the lower panel of Fig. S2, we show the three roots $q_{1,2,3}^{\prime}$ for the case when $q_{\text {comin }}<q<q_{\max }$ which is within the ascending multi-stream regime " $\mathbf{A}_{\mathbf{1}}$ " (see Fig. 1), where

$q_{\min / \max }= \pm \sqrt{2(1-1 / \tau)}-8 c(1-1 / \tau)+O\left(c^{2}\right)$

$q_{\mathrm{comin} / \operatorname{comax}}=\mp \sqrt{8(1-1 / \tau)}-20 c(1-1 / \tau)+O\left(c^{2}\right)$.

The values (B15) can be determined as follows. Firstly, $q_{\min / \max }$ are the locations in the multi-stream regime where the map $x_{\mathrm{ZA}}$ is minimal/maximal. Since the multi-streaming region is very narrow around $q=0$, normalform considerations are sufficient to determine $q_{\min / \max }$. Demanding that $\partial_{q}\left(q+\tau\left[-q+q^{3} / 6+c q^{4}\right]\right) \stackrel{!}{=} 0$, and solving it with perturbation theory then leads to the above reported $q_{\min / \max }$. Secondly, $q_{\text {comin/comax }}$ for which $x_{\mathrm{ZA}}\left(q_{\text {comin/comax }}\right)=x_{\mathrm{ZA}}\left(q_{\min / \max }\right)$ by definition, mark the locations where the flow changes from single- to multi-stream; its values can be determined by demanding that the discriminant of the reduced polynomial (i.e., the square bracketed term in equation B11) vanishes.

Using this, one straightforwardly obtains

$S_{\mathrm{ZA}, 2}=\pi+\left\{\begin{array}{lr}0 ; & q<q_{\mathrm{comin}} \wedge q>q_{\mathrm{comax}} \\ -\operatorname{sign}(q) \sqrt{D(q, \tau)} ; & q_{\mathrm{comin} / \min }<q<q_{\max / \operatorname{comax}} \\ -3 q-36 c(1-1 / \tau) ; & q_{\max }<q<q_{\min }\end{array}\right.$

Summing up, one obtains the integrated multi-streaming force $S_{\mathrm{ZA}}=S_{\mathrm{ZA}, 1}+$ $S_{\mathrm{ZA}, 2}$, i.e.,

$S_{\mathrm{ZA}}=36 c(1-1 / \tau) \Theta(\tau-1)$

$$
+\left\{\begin{array}{lrr}
0 ; & 0 \leq \tau \leq \tau_{1} ; & \mathbf{S}_{\mathbf{1 , 2}} \\
-\operatorname{sign}(q) \sqrt{D(q, \tau)} ; & \tau_{1} \leq \tau \leq \tau_{2} ; & \mathbf{A}_{\mathbf{1 , 2}} \\
-3 q-36 c(1-1 / \tau) ; & \tau \geq \tau_{2}, & \mathbf{B}_{\mathbf{1}, 2}
\end{array}\right.
$$

to first order in $c$, where

$\tau_{1}=8 /\left(8-q^{2}-5 c q^{3}\right), \quad \tau_{2}=2 /\left(2-q^{2}-8 c q^{3}\right)$

are the inverted functions of $q_{\text {comin/comax }}(\tau)$ and $q_{\min / \max }(\tau)$, respectively.

Finally, having determined $S_{\mathrm{ZA}}$ allows us to derive $F\left(x_{\mathrm{ZA}}(q, \tau)\right)=$ $\partial_{q} S_{\mathrm{ZA}}$ which we report here for future reference, we find

$F\left(x_{\mathrm{ZA}}(q, \tau)\right)=\left\{\begin{array}{lr}0 ; & q<q_{\mathrm{comin}} \wedge q>q_{\mathrm{comax}} \\ \frac{A(q) \operatorname{sign}(q)}{\sqrt{D(q, \tau)} ;} & q_{\mathrm{comin} / \min }<q<q_{\max / \text { comax }} \\ -3 ; & q_{\max }<q<q_{\min }\end{array}\right.$

to first order in $c$, where we have defined $A(q):=3 q-36 c\left(1-q^{2}-1 / \tau\right)$. This force is shown in the central panel of Fig. 1 shortly after the first shellcrossing. Observe the slight shift of the force along the horizontal axis in the presence of small but nonzero $c$, breaking the spatial axis-symmetric configuration. This symmetry breaking is precisely the driver for the boost $\xi_{\mathrm{c}}$ to emerge.

\section{B2 Post-shell-crossing displacement}

Once the integrated force $S_{\mathrm{ZA}}$ and the boost $\xi_{\mathrm{c}}$ are determined, the postshell-crossing displacement $\xi$ PSC can be obtained from

$\Re_{\tau} \xi \mathrm{PSC}=-\frac{3}{2} S_{\mathrm{ZA}}$.

This is an inhomogeneous differential equation and can be solved by the method of variation of constants. For this observe that the homogeneous equation $\Re_{\tau} \xi_{\text {PSC }}=0$ has the general solution $\xi_{\text {PSC }}=\lambda \tau+\mu \tau^{-3 / 2}$, where in the homogeneous case, $\lambda$ and $\mu$ are purely spatial. In the inhomogeneous case, these constants are allowed to depend also on time, i.e.,

$\xi_{\mathrm{PSC}}=\lambda(\tau) \tau+\mu(\tau) \tau^{-3 / 2}$,

which we supplement with the constraint

$\dot{\lambda} \tau+\dot{\mu} \tau^{-3 / 2}=0$,

together with the boundary conditions provided at the time of first shellcrossing,

$\xi_{\mathrm{PSC}}(\tau=1)=v^{(\text {ini })}, \quad \dot{\xi}_{\mathrm{PSC}}(\tau=1)=v^{(\text {ini })}$,

where $v^{(\text {ini) }}$ is given in equation (7). To proceed, we plug (B21) into equation (B20), leading to

$\tau^{2} \dot{\lambda}-\frac{3}{2} \dot{\mu} \tau^{-1 / 2}=-\frac{3}{2} S_{\mathrm{ZA}}$.

This equation together with the constraint (B22) form a closed set of equations for $\dot{\lambda}$ and $\dot{\mu}$; integrating the resulting expressions in time from 1 to $\tau$, and using the boundary conditions (B23), we find

$\lambda(\tau, q)=v^{(\text {ini })}-\frac{3}{5} \int_{1}^{\tau} \frac{S_{\mathrm{ZA}}(\eta)}{\eta^{2}} \mathrm{~d} \eta$,

$\mu(\tau, q)=\frac{3}{5} \int_{1}^{\tau} \eta^{1 / 2} S_{\mathrm{ZA}}(\eta) \mathrm{d} \eta$.

Together with the explicit solution for $S_{\mathrm{ZA}}$ given in equation (B17), it is straightforward yet tedious to integrate the expressions (B25), which then leads to $\xi_{\text {PSC }}$ as shown in equation (18a). For this we remark that the first term on the right-hand-side of equation (B17) has no contribution to $\xi_{\text {PSC }}(q)$ for $q \neq 0$, as can be easily verified by employing the center-of-mass condition (3).

\section{SUPPLEMENTARY MATERIAL C: EXPANSION SCHEME AND HIGHER-ORDER FORCE}

In Appendix C1, we provide some details about the employed post-shellcrossing expansion scheme. Then, in Appendix C2, we employ the postshell-crossing results from the main text in order to determine the multistreaming force $F$ in the next ("post-post") iteration.

\section{C1 Expansion scheme}

For convenience let us repeat the evolution equation for the displacement field which is

$\partial_{q} \Re_{\tau} \xi=-\frac{3}{2} F(x(q, \tau))$,

where $\mathfrak{R}_{\tau}=\tau^{2} \partial_{\tau}^{2}+(3 \tau / 2) \partial_{\tau}-3 / 2$, and

$F(x(q, \tau))=\int \partial_{q} \Theta\left[x(q, \tau)-x\left(q^{\prime}, \tau\right)\right] \mathrm{d} q^{\prime}-1$

is the multi-streaming force. To solve equation $(\mathrm{C} 1)$ we employ an iterative expansion scheme in which the ODE of the $n$ th-order approximation of the displacement, here denoted with $\xi_{n}$, is sourced by the multi-streaming force 


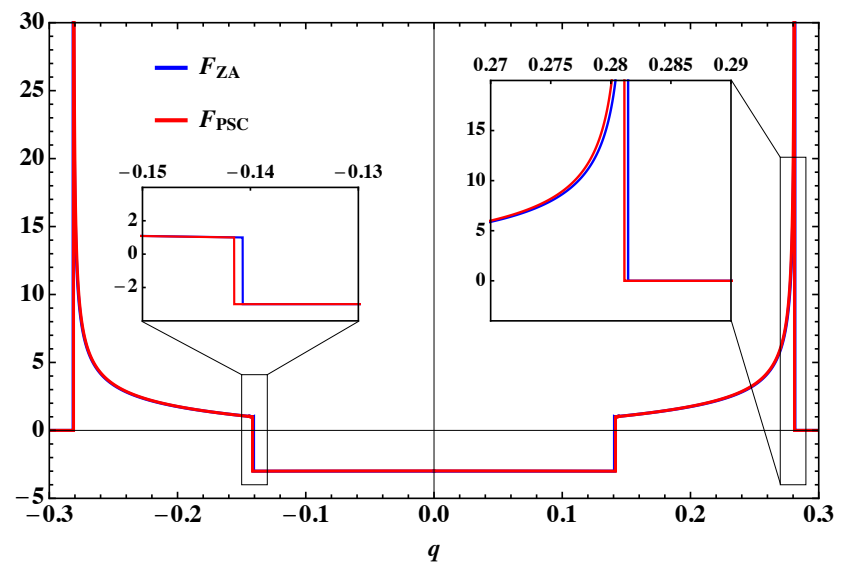

Figure S3. Multi-streaming force within our expansion scheme, at $\tau=$ 1.01, shortly after the first shell-crossing. The blue line denotes the force within the ZA, while the red line is the updated force at the PSC refinement level. For simplicity, we consider the symmetric case $(c=0)$, i.e., we have $F_{\mathrm{ZA}}(q)=F_{\mathrm{ZA}}(-q)$ and equivalently for the PSC force.

that is determined from the displacement at order $n-1$. Thus, in our iterative expansion scheme we solve for

$\partial_{q} \Re_{\tau} \xi \mathrm{PSC} \simeq-\frac{3}{2} F_{\mathrm{ZA}}$,
$\partial_{q} \Re_{\tau} \xi \mathrm{PPSC} \simeq-\frac{3}{2} F_{\mathrm{PSC}}$,
$\partial_{q} \Re_{\tau} \xi \mathrm{PPPSC} \simeq-\frac{3}{2} F_{\mathrm{PPSC}}$,

and so on, where "PSC", "PPSC", etc. denote subsequent higher-order refinements within the post-shell-crossing calculation, and we have introduced the shorthand notation

$F_{\mathrm{ZA}}=F\left(x_{\mathrm{ZA}}(q, \tau)\right), \quad F_{\mathrm{PSC}}=F\left(x_{\mathrm{PSC}}(q, \tau)\right), \quad$ etc.

We remark that our expansion scheme differs from that of Taruya \& Colombi (2017); specifically they suggest to incorporate higher-order refinements by temporal and/or spatial expansions without using (C3). More details to the different approaches used in the literature are provided in App. D.

\section{C2 Force in the post-post-shell-crossing approximation}

Since we have already determined the displacement within the first refinement level in the main text, i.e., $\xi_{\text {PSC }}$ (see equation 18a), we can use this result to determine the force for the next refinement level. Such avenues are an important consistency check: If higher-order corrections to the displacement are not remaining small, it could indicate that the proposed expansion scheme is ill-defined.

Specifically, in our expansion scheme the post-post-shell-crossing displacement $\xi_{\text {PPSC }}$ is governed by equation $(\mathrm{C} 3 \mathrm{~b})$, which requires the multistreaming force $F_{\mathrm{PSC}}$ as input. In Fig. S3 we show this updated force (red lines), together with the Zel'dovich force (blue lines), at times shortly after shell-crossing $\tau=1.01$ for the $c=0$ case of "symmetric" initial data (7). The overall shape of the force is essentially unchanged when going to the higher-order refinement level, however we notice a slight shift of the pronounced features in the force. These shifts are due to the slight change of the spatial locations of $q_{\min / \max }$ and $q_{\text {comin/comax }}$, which can be determined similarly as outlined in Section B1. Specifically, to get $q_{\min / \max }$ one evaluates $\partial_{q}(q+\xi$ PSC $) \stackrel{!}{=} 0$ in perturbation theory, while to get $q_{\text {comin/comax }}$ one needs to find the positions $q^{\prime}$ where the discriminant of the reduced polynomial $\left[x_{\mathrm{PSC}}(q, \tau)-x_{\mathrm{PSC}}\left(q^{\prime}, \tau\right)\right] /\left(q-q^{\prime}\right)$ vanishes. More specific details about higher-order iterations beyond shell-crossing will be assessed in future work.

\section{SUPPLEMENTARY MATERIAL D: COMPARISON OF ANALYTIC DERIVATIONS WITH OTHERS IN THE LITERATURE}

As mentioned in the main text, there exist already a few post-collapse approaches in the literature (Colombi 2015; Taruya \& Colombi 2017; Pietroni 2018). Here we provide some details to the differences and what the approaches have in common.

In doing so we distinguish between approaches that employ initial data without and with symmetry breaking terms (the $c$ term in our notation; cf. equation 7). Of course, the approaches that incorporate symmetry breaking terms have the potential to resolve the resulting non-analyticity at shellcrossing (a.k.a. the boost)

Post-shell-crossing approaches in the $c=0$ case. The cosmological approach of Taruya \& Colombi (2017), itself based on the pioneering work of Colombi (2015) in a non-cosmological setup, introduced the first analytical approach for determining the post-shell-crossing dynamics. Similarly to our approach, Taruya \& Colombi (2017) developed the techniques in 1D, although their approach should be scalable to 3D in a similar way as ours.

The central part of post-shell-crossing studies is determining the effective (multi-streaming) force, for which Taruya \& Colombi (2017) solve the Poisson equation in a periodic box using a Green's method, taking the Ewald summation of periodic repetitions into account. To determine the density in this method one needs to perform a spatial integration over the Green's function times the density; the density is determined using the normal form of the Zel'dovich displacement (a cubic polynomial in $q$; cf. equation A6, or their equation 28). Some of the involved spatial integrals, however, need to be approximated by suitable Taylor expansions. By contrast, in our approach, we determine the Zel'dovich density directly by using the Heaviside step-function, which can be easily integrated over, once the roots of its argument are determined. We remark that the approach of Pietroni (2018) (applied to a 1D cosmology) also exploits Heaviside step-functions, which are then used as "force input" to solve the underlying evolution equation by numerical integration.

Apart from the different force computation of Taruya \& Colombi (2017), there are more differences as regards to solving the underlying evolution equations. While our expansion scheme solves in the first iteration

$\partial_{q}\left[\tau^{2} \partial_{\tau}^{2}+\frac{3 \tau}{2} \partial_{\tau}-3 / 2\right] \xi_{\mathrm{PSC}}=-\frac{3}{2} F_{\mathrm{ZA}}$

with $F_{\mathrm{ZA}}=F\left(x_{\mathrm{ZA}}(q, \tau)\right)$, Taruya \& Colombi (2017) solve instead

$\partial_{q}\left[\tau^{2} \partial_{\tau}^{2}+\frac{3 \tau}{2} \partial_{\tau}\right] \tilde{\xi}_{\mathrm{PSC}}=-\frac{3}{2}\left(F_{\mathrm{ZA}}-\partial_{q} \xi_{\mathrm{ZA}}\right)$

in our notation, where we denote the resulting PSC displacement of Taruya \& Colombi (2017) with $\tilde{\xi}_{\text {PSC }}$. As a consequence, the expansion schemes are fundamentally different, however most likely they will asymptote to the same (convergent) answer at very large orders. The truncation errors at low orders, however, could be substantially different and should be assessed in future work. Nonetheless, the approach of Taruya \& Colombi (2017) appears to be suitable to identify all singularities, but only for the symmetric collapse $(c=0)$.

We remark that $F_{\mathrm{ZA}}=0$ in single-stream regions, thus the evolution equation (D1) reduces to the standard linear ODE for the ZA displacement with the temporal operator $\mathfrak{R}_{\tau}=\tau^{2} \partial_{\tau}^{2}+(3 \tau / 2) \partial_{\tau}-3 / 2$, which has eigenvalues +1 and $-3 / 2$, thus leading to the standard growing $\left(\propto \tau^{+1}\right)$ and decaying-mode $\left(\propto \tau^{-3 / 2}\right)$ solutions. This identification allows us to solve equation (D1) by applying the method of variation of constants, which involves only a single temporal integration (see App. B2 for details). By contrast, in the work of Taruya \& Colombi (2017), two temporal integrations are performed, for which the authors impose further Taylor expansions.

Finally, we note that the instructions of Taruya \& Colombi (2017) for higher-order refinements differ from ours. Specifically, Taruya \& Colombi (2017) suggest potential improvements that involve higher Taylor approximations (in time and space) of the positions $q_{\min / \max }$ and $q_{\text {comin/comax }}$ (cf. Fig. 1), and/or refining some integral expressions that previously were truncated at the leading order in a temporal expansion. By contrast, our scheme 


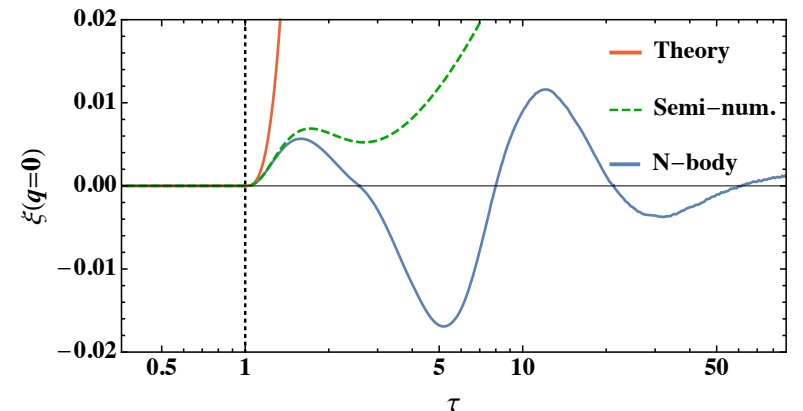

Figure S4. Evolution of the particle that is initially at position $q=0$, as predicted in leading-order theory (red line), by semi-numerical avenues using suitable approximations (green dashed line; see around equation E1), and from high-resolution N-body simulations (blue line). We remark that the shown times are well after the first shell-crossing (indicated by a vertical dotted line in black; the second shell-crossing occurs at $\tau \simeq 2.4$ ). The asymmetry parameter in the initial conditions is $c=0.1$, and for the simulations we used $10^{5} \mathrm{~N}$-body particles and $10^{5}$ time steps.

bootstraps the higher-order displacement to the force computation from the previous order (see equations C3), which is naturally accompanied by a shift of $q_{\min / \max }$ and $q_{\text {comin/comax }}$ at increasing orders; see Fig. S3 and App. C2 for details.

Post-shell-crossing approaches in the $c \neq 0$ case. Although the present implementation of the approach of Taruya \& Colombi (2017) only handles a perfect symmetric collapse, it appears to us that only a few additions are required to generalize it to the asymmetric collapse. For this note that in their Green's approach an integration constant has been set to zero stemming from neglecting the $k=0$ Fourier mode when solving for the Poisson equation (their equation A5). In the asymmetric case, this integration constant takes generally nonzero values after shell-crossing and thus should be restored. Next, one needs to determine the force in the presence of asymmetries in the initial conditions, which we parametrize with $c$; for small $c$, the updated force can be determined using perturbation theory (see App. B1). Once the force is updated accordingly, the integration constant $\xi_{\mathrm{c}}(\tau)$ can be fixed along the lines as discussed in the main text (see around equation 16).

Finally, the approach of Pietroni (2018) solves the 1D Vlasov-Poisson equations (1) together with the Dirac-delta expression for the density (equation 2) by direct numerical integration. They also consider the case of adding an asymmetric distortion at the level of the initial conditions (called "the feature" in Pietroni 2018). While this general strategy is analogous to our $c \neq 0$ case, their initial conditions used rely on an assumed Gaussian shape. Furthermore, to avoid trivial violations of the center-of-mass condition (3), Pietroni (2018) is not adding spatial counter terms in the initial conditions, but instead adjusting a space-independent constant. As a consequence, their (numerical) results are not directly comparable to ours.

\section{SUPPLEMENTARY MATERIAL E: LATE-TIME RESULTS}

\section{E1 Late-time behaviour of the boost}

As we have seen the boost $\xi_{\mathrm{c}}(\tau)$ appears at shell-crossing when the collapse is not perfectly symmetric (parametrized with the asymmetry parameter $c$ ). One consequence of this boost is that the particle that is initially at the position $x=q=0$ begins moving after the first shell-crossing. From previous considerations (see especially Appendix B1) the evolution equation of the particle that is initially at $q=0$ is, exactly and at all times,

$\Re_{\tau} \xi_{\mathrm{c}}=\frac{3}{2}\left[\pi-\int \Theta\left(-x\left(q^{\prime}, \tau\right)\right) \mathrm{d} q^{\prime}\right]$,

where the factor of $\pi$ is a boundary term that can be read off from equation (B10). Figure S4 shows the late-time behaviour of that particle as
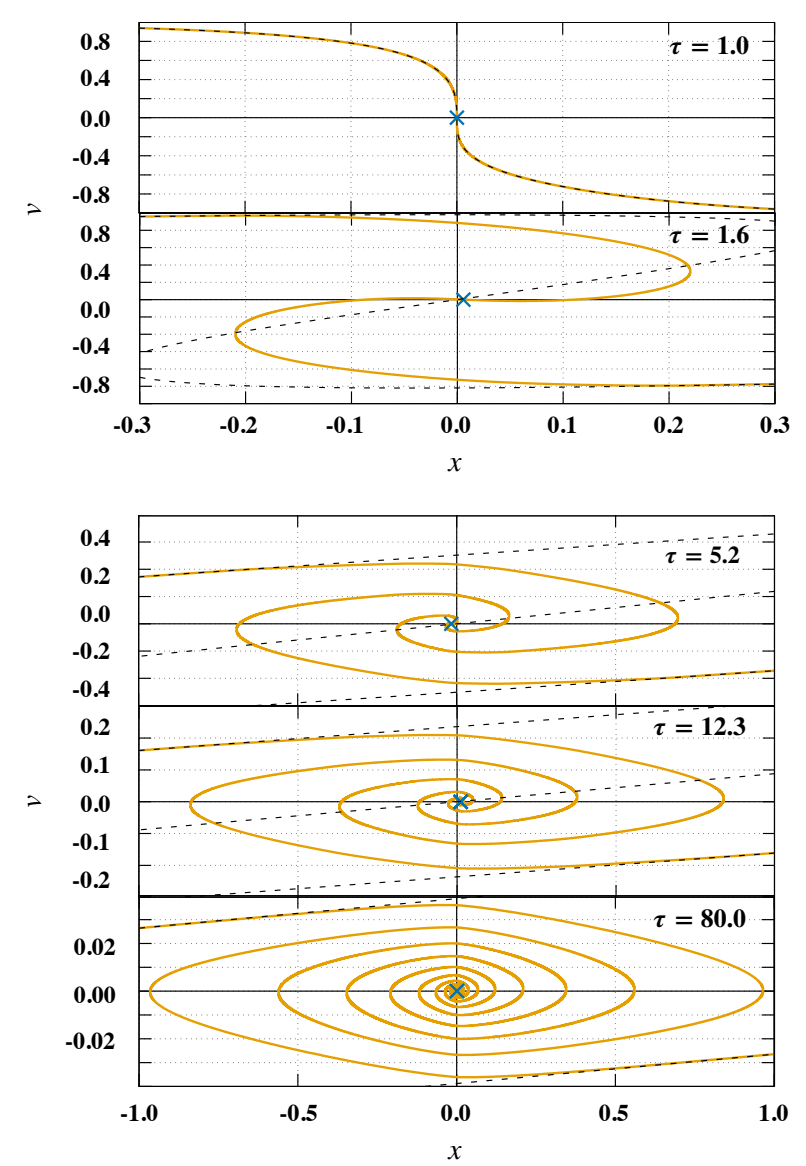

Figure S5. Numerical solution (solid orange lines) of the phase-space for various times in the case of $c=0.1$, using $10^{5} \mathrm{~N}$-body particles and $10^{5}$ time steps. The blue cross denotes the phase-space position of the particle that is at $x=0$ until shell-crossing $(\tau=1)$, which then begins moving due to the nontrivial forcing asymmetry (see also Fig. S4). The black dashed lines denote results in the ZA. Note the different axes scalings employed.

predicted from the leading-order theory (red line; see equation 17), from high-resolution numerical simulations (blue line), as well as by numerically integrating equation (E1) with the replacement $x\left(q^{\prime}, \tau\right) \rightarrow x_{\mathrm{ZA}}\left(q^{\prime}, \tau\right)$ on its RHS (green dashed line). We remark that for the two latter avenues we use $v^{\text {(ini) }}$ as given in equation (7), while the leading-order theory exploits normal-form considerations (using equation $\mathrm{A} 7$ for $v^{(\mathrm{ini})}$ ).

From Fig. S4 it is clear, that the leading-order theory can not encapsulate the non-trivial late-time evolution as observed in the simulation. This is not surprising, as the underlying (analytical) computation is expected to be only meaningful shortly after the first shell-crossing; higher-order refinements as well as theoretical extensions beyond the second shell-crossing are likely to change the conclusions significantly. We keep such avenues for future investigations.

In Fig. S5 we show the corresponding phase-space for various times, where the current position of the fluid particle with label $q=0$ is marked with a blue cross (i.e., the corresponding spatial positions coincide with those from Fig. S4 at the respective times). Similarly as in the previous figure, we observe a damped oscillating behaviour of that particle for sufficiently late times; only at very late times $\tau>1000$, we find that the absolute deviation from zero of this particle is less than $10^{-4}$ (not shown). Naturally, in the limit $\tau \rightarrow \infty$ one would expect that $\xi(q=0) \rightarrow 0$, which is however difficult to achieve in numerical implementations. We keep the study of such attractors for future work. 


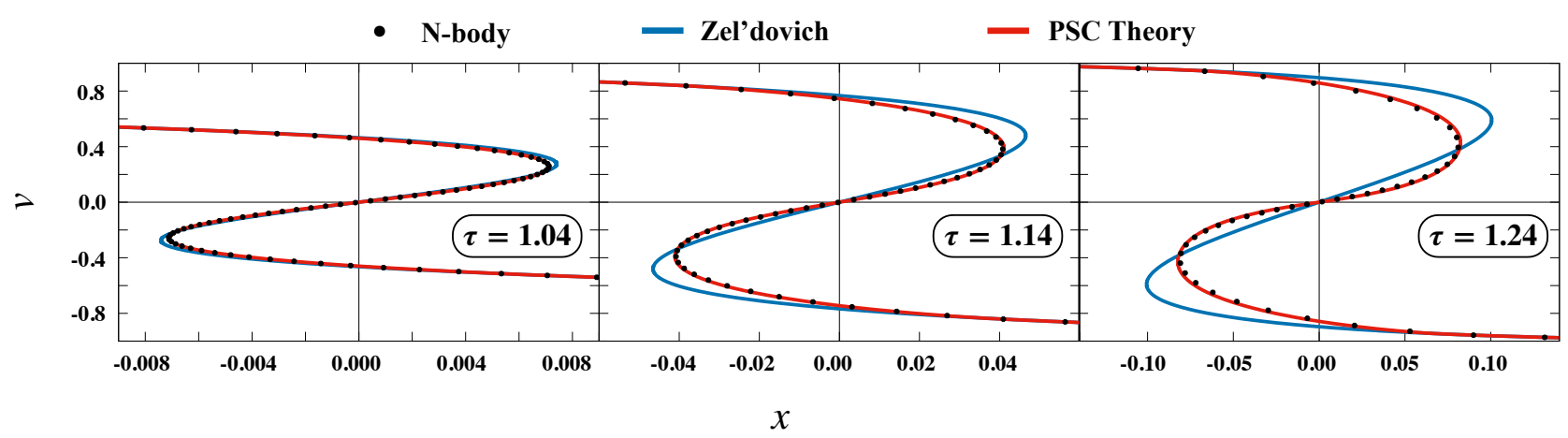

Figure S6. Late-time results in ZA and PSC theory shown respectively in red and blue solid lines, compared against results from our numerical simulations (using 8192 particles/time steps) indicated with black dots. For reasons of better visibility, only a reduced amount of data points from the simulation is shown (left to right panel: every 30th, 50th and 80th data point). Here we have set $c=0$ and, as before, computations are performed on the torus with a period $x \in[-\pi, \pi]$ (i.e., only the interesting parts of the whole phase-space are shown).

\section{E2 Comparison between ZA and PSC theory}

For completeness, we added the ZA prediction in Fig. S5, shown as black dashed lines. Clearly, the ZA is unable to predict the two essential features in the multi-stream regime, namely (1) the development of confined multistream regions (which is well known, see e.g. Taruya \& Colombi 2017), and (2) the non-trivial (right/left) shift of the overall multi-streaming region due to the force imbalance, inherent to asymmetric collapse scenarios $(c \neq 0)$ which, as we elucidate in the main text, has not been investigated before in the literature.

Finally, to eludicate the aforementioned feature (1) further, we show in Fig. S6 a comparison between ZA (blue solid line), PSC theory (red solid line) and simulations (black dots) at late times for the case of $c=0$ (note the different $x$-scalings employed in the panels). While the results from the PSC theory agree reasonably well with those from the numerical simulations until $\tau \lesssim 1.24$, the ZA exemplifies the common overshooting problem, i.e., it lacks the ability for predicting secondary gravitational infall. We remark that this inability of the ZA is well documented in the literature; for recent indepth comparisons at (even) later times between ZA and post-shell-crossing theories/simulations, see Taruya \& Colombi (2017); Pietroni (2018). 